\title{
Lapse function for maximally sliced Brill-Lindquist initial data
}

\author{
Piotr Jaranowski \\ Institute of Theoretical Physics, University of Biatystok \\ Lipowa 41, 15-424 Biatystok, Poland* \\ Gerhard Schäfer \\ Theoretisch-Physikalisches Institut, Friedrich-Schiller-Universität \\ Max-Wien-Platz 1, 07743 Jena, Germany ${ }^{\dagger}$
}

\begin{abstract}
For binary black holes the lapse function corresponding to the Brill-Lindquist initial value solution for uncharged black holes is given in analytic form under the maximal slicing condition. In the limiting case of very small ratio of mass to separation between the black holes the surface defined by the zero value of the lapse function coincides with the minimal surfaces around the singularities.
\end{abstract}

PACS number(s): 04.70.Bw, 04.20.Ex, 04.20.Fy

In a recent paper [1], the authors re-obtained the Brill-Lindquist initial value solution for two electrically uncharged black holes [2] using a point-mass model for the black holes followed by Hadamard's "partie finie" regularization procedure in 3-dimensional space. (It can be shown that the same result appears by applying the dimensional regularization of Ref. [3].) In the present paper we supplement this result by the explicit calculation of the initial lapse function. By the time-symmetry assumption in the Brill-Lindquist initial value solution, the vanishing of the initial shift function automatically occurs. Therefore, all initial value metric coefficients will be known. Our result, for the first time, allows the determination of the motion of test fields and particles near the initial hypersurface for time scales which are small against the time scale of the motion of the two black holes. Similarly, the result will allow discussions of quantum gravitational problems near the initial hypersurface. Furthermore, the knowledge of the initial 4-metric is a step towards an (approximate) analytic treatment of the motion of two black holes starting from Brill-Lindquist initial value data. Finally, numerical head-on-collision simulations starting from those initial data may compare the numerically obtained initial lapse function with the analytic one of the present paper to check the accuracy of the numerical code. In our brief report, we additionally will address the question of how the surface of vanishing initial lapse function, which is the surface of infinite redshift for observers momentarily at rest in the initial hypersurface, is related in some limiting case to the minimal 2-surfaces associated with the Einstein-Rosen bridges of the Brill-Lindquist 3-geometry (see Ref. [2]), knowing that for the Schwarzschild black hole geometry the both types of surfaces coincide (see, e.g., Ref. 佰).

We use units in which $16 \pi G=c=1$, where $G$ is the Newtonian gravitational constant and $c$ is the velocity of light. In the paper all vectors and their lengths are defined in the 3-dimensional Euclidean space endowed with a standard Euclidean metric; $\mathbf{x}$ is the position of an arbitrary point in this space.

In the generalized isotropic ADM gauge [see Eqs. (7-4.22) and (7-4.23) in Ref. [5]] the 3-metric takes the form

$$
g_{i j} \equiv\left(1+\frac{1}{8} \phi\right)^{4} \delta_{i j}+h_{i j}^{\mathrm{TT}}
$$

and for the canonical conjugate $\pi^{i j}$ of the 3-metric, which in terms of the extrinsic curvature $K_{i j}$ of the spacelike hypersurfaces $t=$ const is given by (see, e.g., Ref. [4]

$$
\pi^{i j}=-g^{1 / 2}\left(g^{i l} g^{j m}-g^{i j} g^{l m}\right) K_{l m},
$$

where $g^{i j}$ is the inverse of $g_{i j}$ and $g \equiv \operatorname{det}\left(g_{i j}\right)$, the condition

$$
\pi^{i i} \equiv 0
$$

holds.

\footnotetext{
*Email address: pio@alpha.uwb.edu.pl

${ }^{\dagger}$ Email address: gos@tpi.uni-jena.de
} 
In case of time-symmetric initial conditions the black-hole linear momenta $p_{a i}(a=1,2)$ and field canonical conjugate $\pi^{i j}$, and thus the shift function, $N_{i}=g_{0 i}$, too, are zero

$$
p_{a i}=0, \quad \pi^{i j}=0, \quad N_{i}=0 .
$$

Conformal flatness, i.e.

$$
g_{i j}=\left(1+\frac{1}{8} \phi\right)^{4} \delta_{i j}
$$

additionally implies the vanishing of the transverse-traceless part of the 3-metric,

$$
h_{i j}^{\mathrm{TT}}=0 .
$$

In this case, the coordinate condition (3) coincides with the maximal slicing condition, i.e., $g^{i j} K_{i j}=0$. Under the above conditions, the lapse function $N_{0}\left(N_{0}^{2}=-g_{00}+g^{i j} N_{i} N_{j}=-g_{00}\right.$, as $\left.N_{i}=0\right)$ fulfills the equation

$$
g^{1 / 2} g^{i j} D_{i} D_{j} N_{0}=\frac{1}{4} N_{0} g^{1 / 2} R,
$$

where $D_{i}$ means the covariant derivative with respect to $g_{i j}$, and $R$ is the intrinsic curvature of the hypersurface $t=$ const. The Eq. (7) is easily obtained from the field equation for $\pi^{i i}$, i.e., from $\partial \pi^{i i} / \partial t \equiv 0$ [see, e.g., Eq. (2.7a) in Ref. [6]].

After substituting the ansatz

$$
N_{0}=\frac{1-\psi / 8}{1+\phi / 8}
$$

into the Eq. (7), we obtain the following equation for the function $\psi$ :

$$
\left(1+\frac{1}{8} \phi\right) \Delta \psi=\left(1-\frac{1}{8} \psi\right) \Delta \phi
$$

To derive Eq. (9) we have employed the relation, valid for application on scalar functions,

$$
g^{1 / 2} g^{i j} D_{i} D_{j}=\frac{\partial}{\partial x^{i}}\left(g^{1 / 2} g^{i j} \frac{\partial}{\partial x^{j}}\right)
$$

and the following formula, valid when $h_{i j}^{\mathrm{TT}}=0$ (see, e.g., Ref. [6]):

$$
g^{1 / 2} R=-\left(1+\frac{1}{8} \phi\right) \Delta \phi
$$

The Brill-Lindquist solution reads, [2],

$$
\phi=8\left(\frac{\alpha_{1}}{r_{1}}+\frac{\alpha_{2}}{r_{2}}\right),
$$

where the parameters $\alpha_{1}$ and $\alpha_{2}$ are constants and where $r_{1}$ and $r_{2}$ denote the coordinate distances between the field point and the black hole positions in the conformally related flat space. The parameters $\alpha_{1}, \alpha_{2}$ are related to the bare masses $m_{1}, m_{2}$ of the two black holes through the formulas (see Appendix A in Ref. [7])

$$
\begin{aligned}
& \alpha_{1}=-\frac{1}{4}\left(2 r_{12}+\frac{m_{2}-m_{1}}{16 \pi}\right)+\frac{1}{4} r_{12} \sqrt{4+\frac{m_{1}+m_{2}}{4 \pi r_{12}}+\left(\frac{m_{1}-m_{2}}{16 \pi r_{12}}\right)^{2}}, \\
& \alpha_{2}=-\frac{1}{4}\left(2 r_{12}+\frac{m_{1}-m_{2}}{16 \pi}\right)+\frac{1}{4} r_{12} \sqrt{4+\frac{m_{1}+m_{2}}{4 \pi r_{12}}+\left(\frac{m_{1}-m_{2}}{16 \pi r_{12}}\right)^{2}},
\end{aligned}
$$

where $r_{12}$ denotes the coordinate distance of the two black holes. 
Equation (9) is invariant against $\psi \rightarrow-\phi, \phi \rightarrow-\psi$, which obviously suggests identical structures of the functions $\phi$ and $\psi$ involved in this equation. We therefore search for a solution of the function $\psi$ of the form

$$
\psi=8\left(\frac{\beta_{1}}{r_{1}}+\frac{\beta_{2}}{r_{2}}\right),
$$

where $\beta_{1}$ and $\beta_{2}$ are some new constants. After substitution Eqs. (10) and (12) into Eq. (9) we make use of the regularization procedure of Ref. [1] (or Ref. [3) which relies here on replacing $f(\mathbf{x}) \delta\left(\mathbf{x}-\mathbf{x}_{a}\right)$ by $f_{\text {reg }}\left(\mathbf{x}_{a}\right) \delta\left(\mathbf{x}-\mathbf{x}_{a}\right)$, where the regularized value $f_{\text {reg }}\left(\mathbf{x}_{a}\right)$ of the function $f$ at its (possibly) singular point $\mathbf{x}=\mathbf{x}_{a}$ we define by means of the Hadamard's "partie finie" procedure. We expand $f\left(\mathbf{x}_{a}+\varepsilon \mathbf{n}\right)$ (where $\mathbf{n}$ is a unit vector and $\varepsilon>0$ is a number) into a Laurent series around $\varepsilon=0$ and as the regularized value of the function $f$ at $\mathbf{x}_{a}$ we take the zero-order coefficient of the series averaged over all directions $\mathbf{n}$ :

$$
f\left(\mathbf{x}_{a}+\varepsilon \mathbf{n}\right)=\sum_{m=-N}^{\infty} a_{m}(\mathbf{n}) \varepsilon^{m}, \quad f_{\mathrm{reg}}\left(\mathbf{x}_{a}\right) \equiv \frac{1}{4 \pi} \oint d \Omega a_{0}(\mathbf{n}) .
$$

The usage of the definition (13) boils down in our computation to simple formulas

$$
\begin{aligned}
& \frac{\delta\left(\mathbf{x}-\mathbf{x}_{1}\right)}{\left|\mathbf{x}-\mathbf{x}_{1}\right|}=0, \quad \frac{\delta\left(\mathbf{x}-\mathbf{x}_{1}\right)}{\left|\mathbf{x}-\mathbf{x}_{2}\right|}=\frac{\delta\left(\mathbf{x}-\mathbf{x}_{1}\right)}{\left|\mathbf{x}_{1}-\mathbf{x}_{2}\right|} \\
& \frac{\delta\left(\mathbf{x}-\mathbf{x}_{2}\right)}{\left|\mathbf{x}-\mathbf{x}_{2}\right|}=0, \quad \frac{\delta\left(\mathbf{x}-\mathbf{x}_{2}\right)}{\left|\mathbf{x}-\mathbf{x}_{1}\right|}=\frac{\delta\left(\mathbf{x}-\mathbf{x}_{2}\right)}{\left|\mathbf{x}_{1}-\mathbf{x}_{2}\right|}
\end{aligned}
$$

Making use of Eqs. 14 we have obtained, by comparing the coefficients at Dirac delta functions, the following algebraic equations for the constants $\beta_{1}$ and $\beta_{2}$ :

$$
\begin{aligned}
& \left(1+\frac{\alpha_{2}}{r_{12}}\right) \beta_{1}=\left(1-\frac{\beta_{2}}{r_{12}}\right) \alpha_{1}, \\
& \left(1+\frac{\alpha_{1}}{r_{12}}\right) \beta_{2}=\left(1-\frac{\beta_{1}}{r_{12}}\right) \alpha_{2} .
\end{aligned}
$$

The unique solution of the both equations is easily obtained. It reads

$$
\begin{aligned}
& \beta_{1}=\alpha_{1} \frac{r_{12}+\alpha_{1}-\alpha_{2}}{r_{12}+\alpha_{1}+\alpha_{2}}, \\
& \beta_{2}=\alpha_{2} \frac{r_{12}+\alpha_{2}-\alpha_{1}}{r_{12}+\alpha_{1}+\alpha_{2}} .
\end{aligned}
$$

In the limit $r_{12} \rightarrow \infty$, where two completely separated Schwarzschild black holes have to arise, it holds: $\beta_{1}=\alpha_{1}$ and $\beta_{2}=\alpha_{2}$. If $\alpha_{1}=0$, then $\beta_{1}=0$ and $\beta_{2}=\alpha_{2}$ (analogously, if $\alpha_{2}=0$, then $\beta_{2}=0$ and $\beta_{1}=\alpha_{1}$ ). Also, if $\alpha_{1}=\alpha_{2}$, then $\beta_{1}=\beta_{2}$. Thus, the solution for $\psi$ does have correct limiting values. Furthermore, the post-Newtonian expansion of our solution for $\psi$ coincides with the corresponding part of the lapse function obtained in Ref. [6] through second post-Newtonian order.

For completeness, we give the initial 4-dimensional line element. It takes the form

$$
d s^{2}=-\left(\frac{1-\psi / 8}{1+\phi / 8}\right)^{2} d t^{2}+(1+\phi / 8)^{4}\left(d x^{2}+d y^{2}+d z^{2}\right),
$$

where $x, y, z$ denote Cartesian coordinates and where the Eqs. (10), (12), and (16) [and also Eqs. (11) if the dependence in terms of bare masses is needed] have to be substituted.

In the case of Schwarzschild black holes (there, $\psi=\phi$ ), the surface $N_{0}=0$ (being there the cross-section of a $t=$ const slice with the event horizon) coincides with the throat of the Einstein-Rosen bridge which is a minimal 2 -surface in 3 -space. For binary black-hole systems, the surfaces $N_{0}=0$ need not to be identical with minimal 2 -surfaces, respectively throats, in 3-space. However, an explicit calculation has shown that the initial value surface $N_{0}=0$ (or, $\psi=8$ ), coincides with the minimal surfaces around the singularities in the limiting case of very large separation between the black holes, i.e., we exactly have reproduced the Eq. (27) in Ref. [2] for uncharged black holes. 
P.J. thanks the Theoretisch-Physikalisches Institut of the FSU Jena for hospitality during the realization of this work. The work of P.J. was supported by the EU Programme "Improving the Human Research Potential and the Socio-Economic Knowledge Base" (Research Training Network Contract HPRN-CT-2000-00137) and by the Polish KBN Grant No. 5 P03B 03420.

[1] P. Jaranowski and G. Schäfer, Phys. Rev. D 61, 064008 (2000).

[2] D. R. Brill and R. W. Lindquist, Phys. Rev. 131, 471 (1963).

[3] T. Damour, P. Jaranowski, and G. Schäfer, Phys. Lett. B 513, 147 (2001).

[4] C. W. Misner, K. S. Thorne, and J. A. Wheeler, Gravitation (Freeman, San Francisco, 1973).

[5] R. Arnowitt, S. Deser, and C. W. Misner, in Gravitation: An Introduction to Current Research, edited by L. Witten (Wiley, New York, 1962), pp. 227-265.

[6] G. Schäfer, Ann. Phys. (N.Y.) 161, 81 (1985).

[7] P. Jaranowski and G. Schäfer, Phys. Rev. D 60, 124003 (1999). 